\title{
Efecto de la valencia afectiva del pensamiento sobre la temperatura nasal: imaginería guiada y estrés psicosocial ${ }^{1}$
}

\section{Effect of affective valence of thinking on nasal temperature: Guided imagery and psychosocial stress}

\author{
David Alberto Rodríguez-Medina², Benjamin Domínguez Trejo², \\ Mariana Ortiz Omaña ${ }^{3}$, Gerardo Leija Alva ${ }^{2,4}$ \\ y Omar Arturo Chavarría Santiago ${ }^{2}$
}

\begin{abstract}
RESUMEN
Durante situaciones desagradables, un reflejo simpático de vasoconstricción en la piel ocasiona una reducción en la temperatura periférica, mientras que estados afectivos agradables promueven su aumento. Una de las estrategias clínicas que promueven un estado afectivo agradable es la imaginería guiada. Sin embargo, sus efectos sobre la temperatura nasal (un área sensible a los cambios emocionales) no han sido estudiados. La presente investigación tuvo como propósito evaluar el efecto de la valencia de un pensamiento agradable (imaginería guiada) y la de un pensamiento desagradable (estrés psicosocial) sobre la temperatura nasal, mediante el Trier Social Stress Test, en una versión cognitiva adaptada para este trabajo (TSST-C). Participaron 33 estudiantes universitarios libres de enfermedad inflamatoria, los cuales se dividieron en dos grupos: uno de imaginería guiada y otro de estrés psicosocial. Se utilizó un termómetro de luz infrarroja para evaluar la temperatura nasal sin contacto con el sujeto. Se compararon las mediciones pre y post durante una sesión de cuatro minutos en cada uno de los grupos. Los resultados indican diferencias estadísticamente significativas antes y después de la inducción del pensamiento (positivo y negativo). Sin embargo, la imaginería guiada logró incrementar significativamente la temperatura nasal de los participantes en ese grupo; mientras que el TSST-C decrementó la temperatura en la gran mayoría de los participantes del otro grupo. Se discuten las implicaciones clínicas de regular la actividad simpática durante situaciones desagradables agudas y crónicas, así como la necesidad de evaluación y de la posible intervención biopsicosocial en aquellos participantes que no lograron incrementar su temperatura nasal; asimismo, se sugiere el uso de dispositivos tecnológicos portátiles sensibles a los procesos psicofisiológicos.
\end{abstract}

Palabras clave: Temperatura nasal; Trier Social Stress Test-Cognitive; Imaginería guiada; Estrés.

\footnotetext{
${ }^{1}$ Proyecto financiado por el PAPIIT, Clave IN304515: "Biomarcadores (autonómicos e inmunológicos) como indicadores del componente emocional en el dolor crónico". Los autores agradecen a la clase de Psicopatología del Dr. Carlos Figueroa López, de la Licenciatura en Psicología Clínica del ITESM, Campus Ciudad de México; al Departamento de Divulgación de Ciencia de la UNAM; a Gabriela Jael Pérez García por su invaluable apoyo; al Grupo de Investigación Mente-Cuerpo de la Facultad de Psicología de la unAM y asociados, y a los Drs. Esael Pineda Sánchez y Erik Mateos Salgado, de la UAEH.

${ }^{2}$ Facultad de Psicología, Universidad Nacional Autónoma de México, Av. Universidad 3004, Col. Copilco-Universidad, Del. Coyoacán, 04510 Ciudad de México, México, correo electrónico: psic.d.rodriguez@comunidad.unam.mx. Artículo recibido el 22 de noviembre de 2017 y aceptado el 23 de enero de 2018.

${ }^{3}$ Facultad de Estudios Superiores Zaragoza, Universidad Nacional Autónoma de México, Av. Guelatao 66, Esq. Czda. Ignacio Zaragoza, Col. Ejército de Oriente, Del. Iztapalapa, 09230 Ciudad de México, México.

${ }^{4}$ Centro Interdisciplinario de Ciencias de la Salud, Instituto Politécnico Nacional Av. de los Maestros, Santo Tomas, Del. Miguel Hidalgo, 11340 Ciudad de México, México.
}

Citación: Rodríguez-Medina, D.A., Domínguez T., B., Ortiz O., M., Leija A., G. y Chavarría S., O.A. (2018). Efecto de la valencia afectiva del pensamiento sobre la temperatura nasal: imaginería guiada y estrés psicosocial. Psicología y Salud, 28(2), 187-194. 


\begin{abstract}
Unpleasant situations lead to a sympathetic reflex of skin vasoconstriction, thus reducing peripheral temperature, while pleasant ones lead to an increase. One clinical strategy that promotes pleasant affective states is guided imagery but its effects on nasal temperature have not been studied. The present study aimed at evaluating the effect of pleasant and unpleasant thoughts (psychosocial stress, through the Trier social stress test, using a Cognitive version adapted for this study: TSST-C) specifically on nasal temperature. Thirty-three university students free of inflammatory disease were recruited and assigned to one of two groups: one was exposed to an induced guided imagery exercise and the other received a psychosocial stress exercise (both were group exercises). An infrared thermometer was used to measure temperature of the nose without actual contact with the subject. Measurements were conducted before and after four-minute sessions, for each group. Results revealed statistically significant differences $(\mathrm{p}<.05)$ before and after thought induction (positive and negative). However, the guided imagery condition increased temperature in $61 \%$ of the participants in that group; while the TSST-C decreased the temperature in $93 \%$ of the participants of the contrasting group. Further research should examine the consequences of the regular clinical implications of sympathetic activity during unpleasant acute and chronic situations, as well as the need for evaluation for viable biopsychosocial intervention in those participants who failed to increase their nasal temperature. Likewise, the use of portable technological devices sensitive to psychophysiological processes is suggested.
\end{abstract}

Key words: Nasal temperature; Trier Social Stress Test-Cognitive; Guided imagery; Stress.

\section{INTRODUCCIÓN}

$\mathrm{E}$ studios recientes han evaluado la actividad autonómica térmica durante la exposición a estímulos de valencia agradable, neutra y desagradable. En estudios hechos en monos, por ejemplo, cuando un simio observa un video o escucha un audio de otro pelear, su temperatura nasal tiende a disminuir. Al término de la exposición del video o audio (estímulo negativo), la temperatura nasal se recupera hasta su estado basal (Kano, Hirata, Deschner, Behringer y Call, 2016). Los estudios revisados por Ioannou, Gallese y Merla (2014) han documentado los cambios de dirección térmica faciales, según los cuales la temperatura nasal disminuye en situaciones de temor o de estrés.

Los estudios hechos en humanos sugieren que la disminución de la temperatura nasal se asocia con la actividad simpática, y que el incremento incita la actividad parasimpática (Panasiti et al., 2016). Salazar et al. (2015) encontraron un resultado similar sobre los cambios autonómicos térmicos faciales, de acuerdo con la valencia del estímulo: la exposición a incentivos positivos aumenta la temperatura nasal, mientras que los negativos la disminuyen. La imagen térmica infrarroja de la Figura 1 muestra el efecto de la exposición de imágenes agradables sobre la temperatura periférica facial (obsérvese el incremento de temperatura en la región nasal y perioral).

Figura 1. Evaluación psicofisiológica térmica facial durante la exposición de imágenes agradables (Fuente: Rodríguez-Medina, Domínguez, Cruz, Morales y Leija, en preparación).

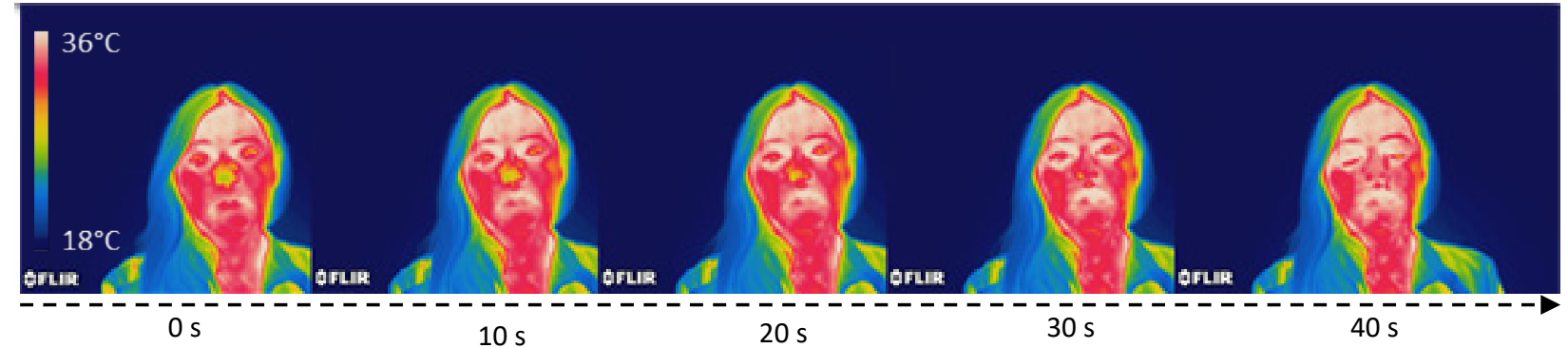

En un estudio piloto, Rodríguez-Medina y colaboradores (2017) reportaron un caso clínico de la actividad vasomotora térmica en los músculos frontales, el cigomático y la punta de la nariz de un paciente con dolor crónico y con entrenamiento en respiración diafragmática durante una tarea 
de priming afectivo. Durante la tarea afectiva, la temperatura nasal decrementó respecto a la línea base, y se incrementó cuando el paciente respiraba diafragmáticamente. Los cambios psicofisiológicos (decremento de temperatura periférica durante situaciones negativas e incremento de temperatura en situaciones agradables) acompañan los cambios clínicos biopsicosociales del paciente sobre los efectos de la intervención psicológica especializada en un problema de dolor crónico. Sin embargo, algunos pacientes no logran beneficiarse de los procedimientos de relajación, como la respiración diafragmática. Estos pacientes -por ejemplo, aquellos con dolor articular- requieren otros procedimientos psicológicos para alcanzar los beneficios terapéuticos de la disminución del tono simpático que está presente durante el dolor y el estrés. Una opción es la imaginería guiada (Baird y Sands, 2006; Bigham, McDannel, Luciano y Salgado-Lopez, 2014; Billquist et al., 2017; Charalambous, Giannakopoulou, Bozas y Paikousis, 2015; Louie, 2004) o el entrenamiento autógeno, cuya validez y efectividad para reducir la actividad simpática se ha documentado en población clínica (Kiba et al., 2015; Kiba et al., 2017) y no clínica (Lim y Kim, 2014). Tales procedimientos modifican la actividad autonómica y endocrinológica asociada al estrés; sin embargo, ninguno de ellos ha evaluado la temperatura de la nariz como indicador de cambio clínico; es decir, el aumento de la actividad parasimpática (Panasiti et al., 2016).

La evidencia disponible sobre el procesamiento cognitivo y la valencia afectiva de los incentivos sugiere que los estímulos positivos o agradables activan circuitos neurales y autonómicos diferentes de los que producen los negativos o desagradables (Allen et al., 2012; Altmann, Bohrn, Lubrich, Menninghaus y Jacobs, 2012; Citron, Gray, Critchley, Weekes y Ferstl, 2014; Nealis, van Allen y Zelenski, 2016). Aun así, se desconoce si la inducción del pensamiento con valencia positiva (imaginería guiada) aumenta la temperatura nasal, y si la inducción del pensamiento con valencia negativa (estresor social cognitivo) la disminuye. En consecuencia, el objetivo de este estudio fue evaluar el efecto de la valencia del pensamiento, inducido por el experimentador, sobre la temperatura nasal en participantes voluntarios clínicamente sanos y libres de enfermedades inflamatorias.

\section{MÉTODO}

\section{Participantes}

Participaron 33 voluntarios, seleccionados mediante un muestreo no probabilístico intencional, divididos en dos grupos: el primero, con 18 participantes (nueve hombres y nueve mujeres), para la inducción del pensamiento con valencia positiva; el segundo, con 15 (dos hombres y trece mujeres) para la inducción del pensamiento con valencia negativa. Todos los participantes eran mayores de edad ( $M=22.53$ años, D.E. \pm 2.72 años), con una escolaridad media de 15.63 años ( \pm 0.68 años), sin entrenamiento en respiración diafragmática, quienes aceptaron ser incluidos en el estudio psicofisiológico térmico como parte de las demostraciones prácticas en su formación profesional.

\section{Materiales}

\section{Non Contact Infrared Body Thermometer DM300}

En estos estudios de investigación clínica y preclínica normalmente se registran los cambios autonómicos mediante sensores adheridos a la piel. Una herramienta tecnológica alternativa es el registro de la temperatura mediante un termómetro de luz infrarroja, el cual tiene la ventaja de ser un equipo barato, portátil y sensible a los cambios autonómicos que no requiere estar en contacto con la piel del sujeto, lo que brinda a este una mayor libertad de movimiento y comodidad.

Este termómetro de luz infrarroja, cuyo díámetro es de $0.5 \mathrm{~cm} 2$, permite cuantificar la temperatura de la superficie de la piel en un rango de $0-100^{\circ} \mathrm{C}$. En el presente estudio se registró la temperatura a una distancia de 3 a $5 \mathrm{~cm}$ de la punta de la nariz.

Trier Social Stress Test (versión modificada cognitiva) (TSST-C).

Es este un paradigma de estrés social propuesto por Kirschbaum, Pirke y Hellhammer (1993). Consiste en cuatro etapas: 1) se aclimata al sujeto en la sala de registro (debe permanecer sentado en reposo durante 15 minutos); 2) uno de los experimentadores le da indicaciones para preparar un discurso con una duración de cinco minutos, en el que el 
participante indica por qué considera que sería un buen candidato para su trabajo ideal; este discurso es videograbado y evaluado por jueces expertos en hablar en público (para preparar el discurso el participante cuenta con tres minutos); 3) el participante presenta su discurso ante un par de evaluadores y videocámaras, y 4) lleva a cabo una numeración regresiva de 17 en 17 a partir de 1021 (para una visualización del protocolo y las indicaciones, véase Birkett [2011]). Este paradigma ha sido ampliamente ultilizado en diversas investigaciones que evalúan los efectos del estrés psicológico en funciones endócrinas, autonómicas, inmunológicas, cognitivas (Allen, Kennedy, Cryan, Dinan y Clarke, 2014), subjetivas (Hellhammer y Schubert, 2012) e incluso genéticas (Allen et al., 2017). Hay algunas versiones modificadas del paradigma (Allen et al., 2017; Johnson et al., 2017; Yim, Quas, Rush, Granger y Skoluda, 2015, entre otras). En este estudio se desarrolló una versión propia, el Trier Social Stress Test-Cognitivo (TSST-C en lo sucesivo), basada en el diseño de von Dawans, Kirschbaum y Heinrichs (2011). Dicha versión constó de las siguientes fases: 1) aclimatación; 2) preparación de escenario, el cual consiste en colocar una videocámara encendida en un escritorio con silla frente al grupo (similar al protocolo original del TSST, aunque en realidad no se graban ni analizan los discursos); 3) distribución de la hoja de indicaciones del TSST, doblada a la mitad para ser abierta al mismo tiempo por todos los participantes, la cual contiene las instrucciones en dos incisos: la preparación del discurso y, en cuanto el instructor lo indique, la tarea aritmética. Por último, en la leyenda final de la hoja se lee lo siguiente:" Tiene cuatro minutos para preparar el discurso y la tarea aritmética para despues pasar a exponerlos frente al grupo. Por favor, cierre los ojos y prepárese". Cumplido lo anterior, el instructor (experimentador) da la señal al grupo para comenzar el ejercicio.

\section{Procedimiento}

Los registros térmicos del grupo que recibió el TSST-C se programaron a las 9 horas, y los del grupo de inducción del pensamiento con valencia positiva a las 12 . Debido a la naturaleza de los registros térmicos, los participantes permanecieron en reposo, sentados, a una temperatura constante de $21^{\circ} \mathrm{C}$. Luego, se les hizo una exposición sobre las medidas psicofisiológicas. Una vez aclimatados a la temperatura de las salas (una por grupo), se procedió al registro térmico; después, se indujo el pensamiento con valencia positiva (indicaciones de imaginería guiada sobre estar en el lugar más cómodo, agradable, cálido, seguro y placentero donde les gustaría estar) o el pensamiento con valencia negativa (aplicación del TSST-C) (Figura 2). Por razones éticas, al grupo que se le indujo el TSST-C se le aplicó la estrategia de relajación.

Figura 2. Diseño de investigación.

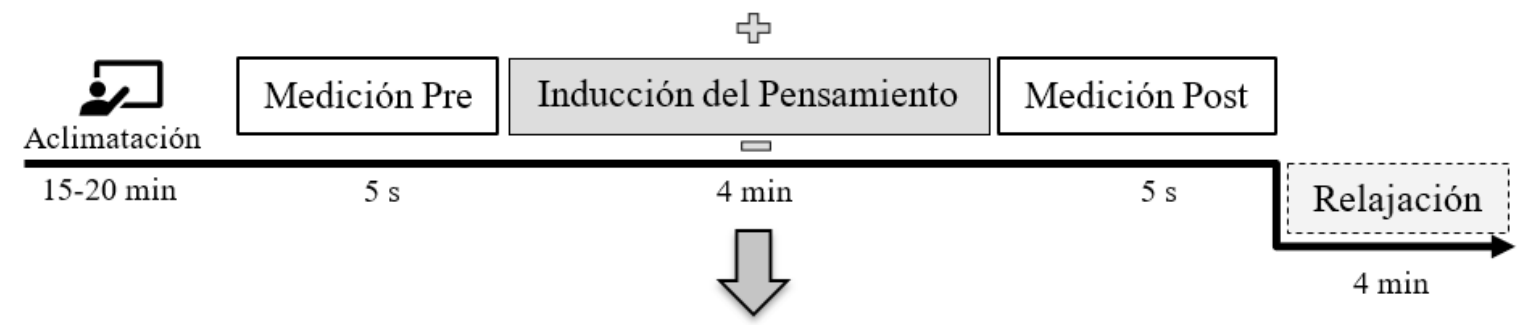

Grupo 1 Valencia Positiva del Pensamiento

Grupo 2 Valencia Negativa del Pensamiento

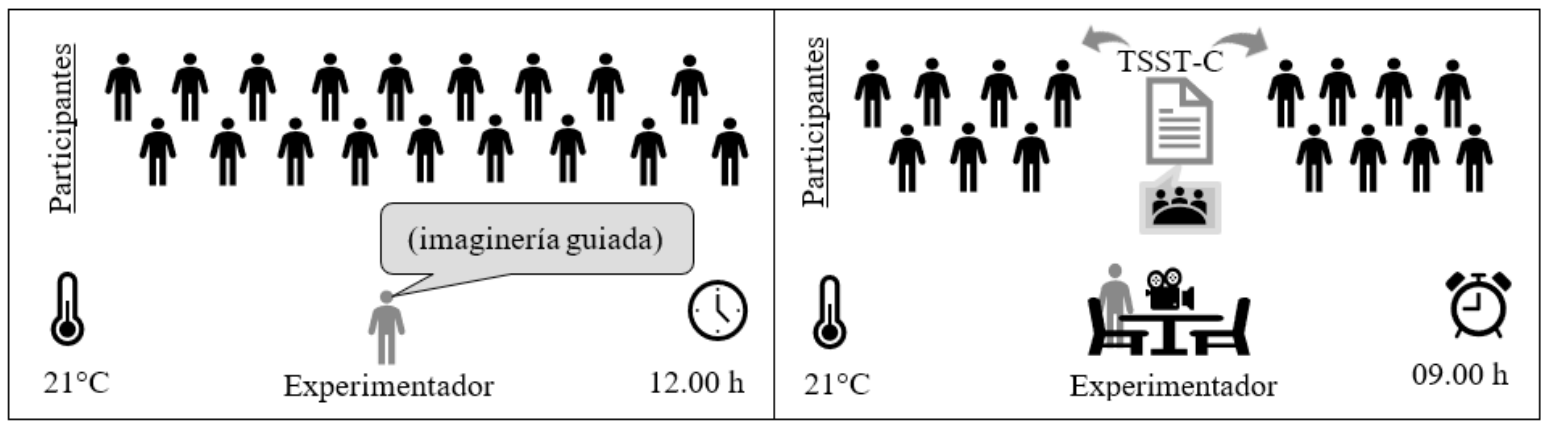


Al grupo 1 se le indujo un pensamiento con valencia positiva (imaginería guiada), con medición pre-post. Al grupo 2 se le indujo el pensamiento con valencia negativa por escrito (TSST-C), y posteriormente a la segunda medición se le aplicó una estrategia de relajación.

\section{Análisis estadísticos}

Se analizó la distribución normal de cada grupo de temperaturas mediante la prueba de Shapiro-Wilk. Debido a que no se encontró normalidad, se procedió a analizar los resultados con estadística no paramétrica. Se comparó el efecto principal pre-postinducción del pensamiento mediante una prueba de Wilcoxon para cada tipo de inducción de pensamiento: con valencia positiva y con valencia negativa. Únicamente para el grupo al que se le administró el TSST-C y luego la relajación se le aplicó la prueba de Friedman. Se extrajeron los porcentajes de sujetos que cambiaron su temperatura nasal en cada tipo de inducción de pensamiento. Finalmente, se compararon las diferencias de temperatura nasal de incrementos y decrementos por cada tipo de inducción mediante la $\mathrm{U}$ de Mann-Whitney. Para las pruebas estadísticas se estableció un nivel de significancia de $p \leq .05$.

\section{RESULTADOS}

Debido a las diferencias de programación de horario entre los grupos, las temperaturas iniciales entre ambos no fueron equivalentes $(p<.001)$. Los resultados de la valencia (positiva y negativa) del pensamiento pre-post se muestran en la Figura 3.

Figura 3. Cambios de temperatura nasal en función de la valencia del pensamiento.

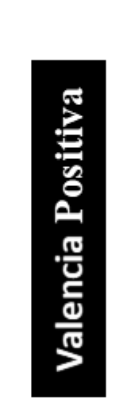

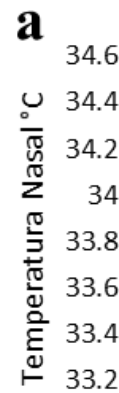
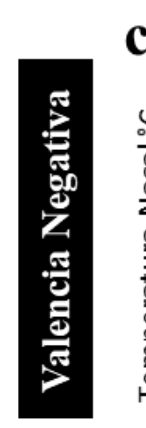

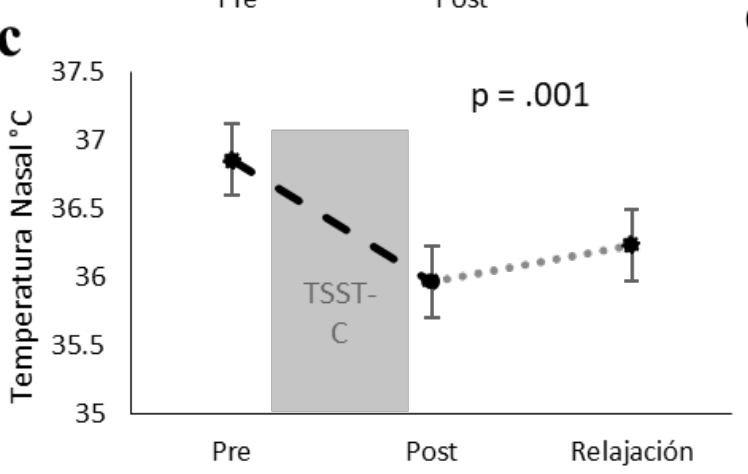

b

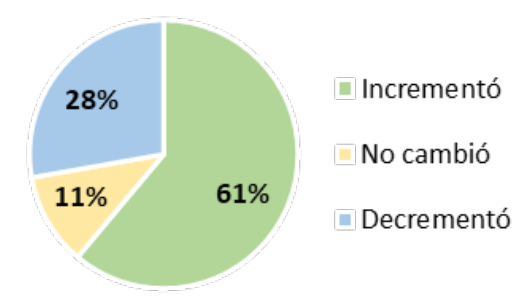

d

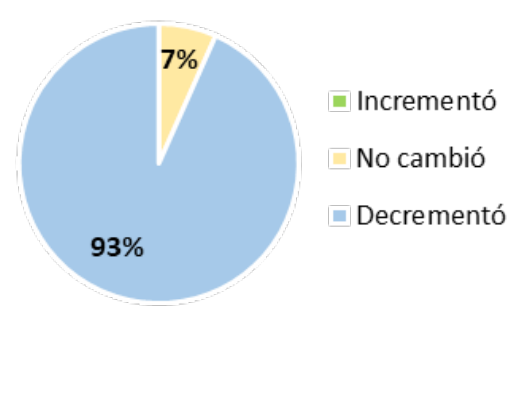

a) El pensamiento con valencia positiva incrementó la temperatura nasal significativamente $(\mathrm{p}<.05)$, b) en porcentaje, la mayoría de los participantes; mientras que c) el grupo al que se le indujo el pensamiento negativo decrementó la temperatura de la nariz $(\mathrm{p}<.05)$ d) en porcentaje, la mayoría de los participantes.

La inducción del pensamiento con valencia positiva aumentó la temperatura nasal significativamente $(\mathrm{Z}=-2.42, p<.05)$. Dicho incremento ocurrió en $61 \%$ de los participantes, mientras que aproxi- madamente $28 \%$ disminuyó su temperatura. Los resultados mostraron una diferencia estadísticamente significativa $(\mathrm{Z}=-3.148, p<.001)$ entre quienes aumentaron su temperatura nasal $\left(\mathrm{M}=0.7^{\circ} \mathrm{C}\right) \mathrm{y}$ 
quienes la disminuyeron con la imaginería guiada $\left(\mathrm{M}=-1^{\circ} \mathrm{C}\right)$.

Por otra parte, la inducción del pensamiento con valencia negativa disminuyó significativamente la temperatura nasal $(\mathrm{Z}=-3.3, p \leq .001)$. Este decremento de la temperatura se encontró en $93 \%$ de los participantes $\left(\mathrm{M}=-0.9^{\circ} \mathrm{C}\right)$, mientras que el 7\% restante no la modificó. Durante el TSST-C ningún participante aumentó su temperatura.

\section{DISCUSIÓN}

El propósito de este estudio fue explorar los incrementos o decrementos de temperatura en función de la valencia afectiva del pensamiento inducido. Similar a otros estímulos afectivos (Salazar et al., 2015), el pensamiento con valencia positiva produjo un incremento de la temperatura nasal -efecto autonómico parasimpático (Panasiti et al., 2016)-, mientras que el pensamiento con valencia negativa generó una disminución de la temperatura nasal -efecto simpático.

Los estudios clínicos del uso de la imaginería guiada han mostrado que es efectiva en la disminución de respuestas cardiovasculares, así como de la tensión muscular (Bigham et al., 2014; Louie, 2004). No obstante, no se había evaluado su efecto sobre la temperatura facial (concretamente de la nariz), la cual es sensible a los cambios afectivos fásicos (Ioannou et al., 2014). Es posible que este aumento de temperatura se deba a los cambios del ciclo respiratorio de mayor espiración respecto a su estado basal de manera pasiva (Lindemann, Leiacker, Rettinger y Keck, 2002), y no activa, como en el entrenamiento en respiración diafragmática, en el que el sujeto debe prestar atención a su respiración cuando se le induce a imaginarse en el lugar más cómodo posible y a percibir las sensaciones agradables de ese lugar. La utilidad clínica de este resultado aporta una evidencia más del uso de esta estrategia terapéutica para disminuir el estrés, que está asociado a una baja temperatura nasal (Ioannou et al., 2014).

A pesar de que los efectos de la imaginería guiada sobre la temperatura nasal fueron estadísticamente significativos de manera grupal, se halló que algunos participantes no lograron aumen- tar su temperatura, mientras que otros incluso la disminuyeron. En tales participantes es necesario evaluar su contexto biopsicosocial (antecedentes familiares de vulnerabilidad al estrés, estresores actuales o recientes que dificulten seguir atentamente las indicaciones de la imaginería guiada, o incluso procesos inflamatorios de enfermedad).

Lo anterior es de especial interés en la práctica psicológica clínica y de la salud debido a la necesidad de evaluar integralmente (evaluación biopsicosocial) los resultados esperados en una población (Rodríguez-Medina et al., 2017). Por ejemplo, un paciente que ha sido víctima de algún estresor traumático potencial, de un trastorno depresivo mayor o de una enfermedad inflamatoria central o periférica (que induce la llamada "conducta de enfermedad") es posible que requiera de otro tipo de atención clínica, siendo una alternativa el manejo de la expresión emocional, por ejemplo. En futuras investigaciones se deben considerar ciertos aspectos biopsicosociales que influyen en el incremento/decremento de la temperatura periférica (hora del día, estado afectivo o, ciclo menstrual, entre otros).

La aplicación del TSST-C como inductor de valencia negativa (estresante) pudo reducir la temperatura nasal en aproximadamente $1^{\circ} \mathrm{C}$. Cuando la temperatura nasal se decrementa en una situación estresante, otras áreas faciales se activan (por ejemplo, el músculo corrugador se contrae) y se inicia una cascada de activación muscular y un aumento de la actividad cardiovascular que son propios del estrés, los que, si se repiten continuamente, facilitan procesos crónicos, como la cefalea tensional o el bruxismo, por ejemplo, o incluso acentúan la sintomatología de cuadros clínicos de dolor crónico. Ello subraya la importancia de controlar el tono simpático mediante cualquier estrategia de relajación que promueva cambios en el ciclo respiratorio, como los ejercicios de respiración diafragmática en aquellos que logran mantener un ritmo continuo de inhalaciones-exhalaciones y pausas prolongadas; la inhalación profunda promueve un incremento de la temperatura nasal y disminuye el estrés subjetivo (Bailey, Casey, Pawar y Garcia, 2017). Para los participantes que fueron expuestos al TSST-C y que no lograron recuperar el nivel de temperatura después de la imaginería guiada se sugiere una reestructuración 
cognitiva, o bien se puede explorar el uso de otra estrategia de relajación, e incluso, si no aumenta, condicionarla con un estímulo térmico externo (temperatura de 30 a $34^{\circ} \mathrm{C}$ ) (Raison, Hale, Williams, Wager y Lowry, 2015) para producir y mantener la vasodilatación.

Un aspecto relevante que debe resaltarse es la utilidad clínica y experimental del TSST. La versión cognitiva que se desarrolló para este estudio, el TSST-C, reduce los tiempos (cuatro minutos en total) y el costo de aplicación (cintas de video), y puede aplicarse de manera grupal; no obstante, requiere que el experimentador disponga de al menos una videocámara y de un grupo de participantes para simular todas las condiciones similares a la TSST grupal (von Dawans et al., 2011).
Finalmente, los estudios de investigación clínica y experimental recurren a equipos de registro con una alta precisión tecnológica, sensibles a los cambios autonómicos; sin embargo, es necesario contar con equipos portátiles de bajo costo que hagan posible cuantificar estos cambios de manera no invasiva ni obstructiva ( paciente), por lo que un termómetro de infrarrojo portátil es útil. Pese a ello, en futuras investigaciones puede emplearse otra herramienta tecnológica de registro térmico más precisa, como la cámara térmica de infrarrojo (Rodríguez-Medina, Albarrán, Trejo, Hernández y Alba, 2018), cuya validez se ha demostrado en la investigación psicofisiológica afectiva (Clay-Warner y Robinson, 2014; Ioannou et al., 2014) y de la tasa respiratoria (Barbosa et al., 2016).

\section{REFERENCIAS}

Allen, M., Dietz, M., Blair, K., van Beek, M., Rees, G., Vestergaard-Poulsen, P., et al. (2012). Cognitive-affective neural plasticity following active-controlled mindfulness intervention. Journal of Neuroscience, 32(44), 15601-15610. Disponible en http:// dx.doi:10.1523/jneurosci.2957-12.2012.

Allen, A., Kennedy, P., Cryan, J., Dinan, T. y Clarke, G. (2014). Biological and psychological markers of stress in humans: Focus on the Trier Social Stress Test. Neuroscience \& Biobehavioral Reviews, 38, 94-124. Disponible en http://dx.doi. org/10.1016/j.neubiorev.2013.11.005.

Allen, A., Kennedy, P., Dockray, S., Cryan, J., Dinan, T. y Clarke, G. (2017). The Trier Social Stress Test: Principles and practice. Neurobiology Of Stress, 6, 113-126. Disponible en http://dx.doi.org/10.1016/j.ynstr.2016.11.001.

Altmann, U., Bohrn, I., Lubrich, O., Menninghaus, W. y Jacobs, A. (2012). The power of emotional valence -from cognitive to affective processes in reading. Frontiers in Human Neuroscience, 6. Disponible en http://dx.doi.org/10.3389/fnhum.2012.00192.

Bailey, R., Casey, K., Pawar, S. y Garcia, G. (2017). Correlation of nasal mucosal temperature with subjective nasal patency in healthy individuals. JAMA Facial Plastic Surgery, 19(1), 46. Disponible en http://dx.doi:10.1001/jamafacial.2016.1445.

Baird, C. y Sands, L. (2006). Effect of guided imagery with relaxation on health-related quality of life in older women with osteoarthritis. Research in Nursing \& Health, 29(5), 442-451. Disponible en http://dx.doi.org/10.1002/nur.20159.

Barbosa P., C., Yu, X., Czaplik, M., Blazek, V., Venema, B. y Leonhardt, S. (2016). Estimation of breathing rate in thermal imaging videos: a pilot study on healthy human subjects. Journal of Clinical Monitoring and Computing. Disponible en http:// dx.doi:10.1007/s10877-016-9949-y.

Bigham, E., McDannel, L., Luciano, I. y Salgado-Lopez, G. (2014). Effect of a brief guided imagery on stress. Biofeedback, 42(1), 28-35. Disponible en http://dx.doi.org/10.5298/1081-5937-42.1.07.

Billquist, E., Michelfelder, A., Brincat, C., Brubaker, L., Fitzgerald, C. y Mueller, E. (2017). Pre-operative guided imagery in female pelvic medicine and reconstructive surgery: a randomized trial. International Urogynecology Journal. Disponible en http://dx.doi.org/10.1007/s00192-017-3443-z.

Birkett, M. (2011). The Trier Social Stress Test Protocol for inducing psychological stress. Journal of Visualized Experiments, 56. Disponible en http://dx.doi:10.3791/3238.

Charalambous, A., Giannakopoulou, M., Bozas, E. y Paikousis, L. (2015). A randomized controlled trial for the effectiveness of progressive muscle relaxation and guided imagery as anxiety reducing interventions in breast and prostate cancer patients undergoing chemotherapy. Evidence-Based Complementary And Alternative Medicine, 2015, 1-10. Disponible en http:// dx.doi.org/10.1155/2015/270876.

Citron, F., Gray, M., Critchley, H., Weekes, B. y Ferstl, E. (2014). Emotional valence and arousal affect reading in an interactive way: Neuroimaging evidence for an approach-withdrawal framework. Neuropsychologia, 56, 79-89. Disponible en http:// dx.doi.org/10.1016/j.neuropsychologia.2014.01.002. 
Clay-Warner, J. y Robinson, D. (2014). Infrared thermography as a measure of emotion response. Emotion Review, 7(2), 157-162. Disponible en http://dx.doi:10.1177/1754073914554783.

Hellhammer, J. y Schubert, M. (2012). The physiological response to Trier Social Stress Test relates to subjective measures of stress during but not before or after the test. Psychoneuroendocrinology, 37(1), 119-124. Disponible en http://dx.doi.org/10.1016/j.psyneuen.2011.05.012.

Ioannou, S., Gallese, V. y Merla, A. (2014). Thermal infrared imaging in psychophysiology: Potentialities and limits. Psychophysiology, 51(10), 951-963. Disponible en http://dx.doi.org/10.1111/psyp.12243.

Johnson, M., Deardorff, J., Parra, K., Alkon, A., Eskenazi, B. y Shirtcliff, E. (2017). A modified Trier Social Stress Test for vulnerable Mexican American adolescents. Journal of Visualized Experiments, 125. Disponible en http://dx.doi.org/10.3791/55393.

Kano, F., Hirata, S., Deschner, T., Behringer, V. y Call, J. (2016). Nasal temperature drop in response to a playback of conspecific fights in chimpanzees: A thermo-imaging study. Physiology \& Behavior, 155, 83-94. http://dx.doi.org/10.1016/j.physbeh.2015.11.029.

Kiba, T., Abe, T., Kanbara, K., Kato, F., Kawashima, S., Saka, Y., et al. (2017). The relationship between salivary amylase and the physical and psychological changes elicited by continuation of autogenic training in patients with functional somatic syndrome. Biopsychosocial Medicine, 11(1). Disponible en http://dx.doi.org/10.1186/s13030-017-0103-y.

Kiba, T., Kanbara, K., Ban, I., Kato, F., Kawashima, S., Saka, Y., Yamamoto, K., Nshiyama, J., Mizuno, Y., Abe, T. y Fukunaga, M. (2015). Saliva amylase as a measure of sympathetic change elicited by autogenic training in patients with functional somatic syndromes. Applied Psychophysiology and Biofeedback, 40(4), 339-347. Disponible en http://dx.doi.org/10.1007/ s10484-015-9303-z.

Kirschbaum, C., Pirke, K.M. y Hellhammer, D.H. (1993). The Trier Social Stress Test: a tool for investigating psychobiological stress responses in a laboratory setting. Neuropsychobiology, 28, 76-81.

Lim, S. y Kim, C. (2014). Effects of autogenic training on stress response and heart rate variability in nursing students. Asian Nursing Research, 8(4), 286-292. Disponible en http://dx.doi.org/10.1016/j.anr.2014.06.003.

Lindemann, J., Leiacker, R., Rettinger, G. y Keck, T. (2002). Nasal mucosal temperature during respiration. Clinical Otolaryngology and Allied Sciences, 27(3), 135-139. Disponible en http://dx.doi::10.1046/j.1365-2273.2002.00544.x.

Louie, S. (2004). The effects of guided imagery relaxation in people with COPD. Occupational Therapy International, 11(3), 145159. Disponible en http://dx.doi.org/10.1002/oti.203.

Nealis, L., van Allen, Z. y Zelenski, J. (2016). Positive affect and cognitive restoration: investigating the role of valence and arousal. PlosOne, 11(1), e0147275. Disponible en http://dx.doi:10.1371/journal.pone.0147275.

Panasiti, M., Cardone, D., Pavone, E., Mancini, A., Merla, A. y Aglioti, S. (2016). Thermal signatures of voluntary deception in ecological conditions. Scientific Reports, 6(1). http://dx.doi.org/10.1038/srep35174.

Raison, C., Hale, M., Williams, L., Wager, T. y Lowry, C. (2015). Somatic influences on subjective well-being and affective disorders: the convergence of thermosensory and central serotonergic systems. Frontiers in Psychology, 5. Disponible en http:// dx.doi.org/10.3389/fpsyg.2014.01580.

Rodríguez-Medina, D., Albarrán, I., Trejo, B., Hernández, L. y Alva, G. (en preparación). Efecto del entrenamiento en reconocimiento y reproducción facial emocional sobre la temperatura facial. México: Universidad Nacional Autónoma de México/ Universidad Autónoma de Querétaro.

Rodríguez-Medina, D., Albarrán, I., Trejo, B., Hernández, L., Alva, G. y Esteban, P. (2017). Psychophysiological facial thermal assessment of the relaxation in a patient with osteoarthrosis. Pan American Journal of Medical Thermology, 3, 33-36. Disponible en http://dx.doi:10.18073/2358-4696/pajmt.v3n1p33-36.

Salazar-López, E., Domínguez, E., Juárez-Ramos, V., De la Fuente, J., Meins, A., Iborra, O., et al. (2015). The mental and subjective skin: Emotion, empathy, feelings and thermography. Consciousness and Cognition, 34, 149-162. Disponible en http:// dx.doi.org/10.1016/j.concog.2015.04.003.

von Dawans, B., Kirschbaum, C. y Heinrichs, M. (2011). The Trier Social Stress Test for Groups (TSST-G): A new research tool for controlled simultaneous social stress exposure in a group format. Psychoneuroendocrinology, 36(4), 514-522. Disponible en http://dx.doi.org/10.1016/j.psyneuen.2010.08.004.

Yim, I., Quas, J., Rush, E., Granger, D. y Skoluda, N. (2015). Experimental manipulation of the Trier Social Stress Test-Modified (TSST-M) to vary arousal across development. Psychoneuroendocrinology, 57, 61-71. Disponible en http://dx.doi. org/10.1016/j.psyneuen.2015.03.021. 